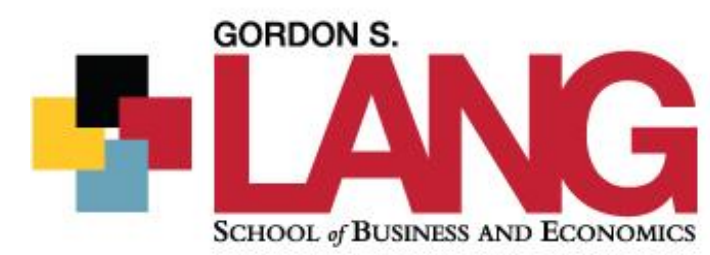

Department of Economics and Finance

Gordon S. Lang School of Business and Economics

University of Guelph

Discussion Paper 2019-01

\title{
Coordination in Closed-loop supply chain with price-dependent returns
}

By:

\author{
Talat Genc \\ University of Guelph \\ tgenc@uoguelph.ca
}

\author{
Pietro De Giovanni \\ ESSEC Business School \\ pietro.degiovanni@essec.edu
}




\title{
Coordination in Closed-loop supply chain with price-dependent
}

\author{
returns
}

\author{
Pietro De Giovanni \\ Department of Operations Management, ESSEC Business School, Paris, France \\ Talat S. Genc \\ Department of Economics, University of Guelph, Guelph, Ontario, Canada
}

18th March 2019

\begin{abstract}
This paper proposes two Closed-loop Supply Chain (CLSC) games in which a manufacturer sets some green activity programs efforts and a retailer sets the selling price. Both strategies influence the return rate, which is a state variable. The pricing strategy plays a key role in the identification of the best contract to achieve coordination as well as in achieving environmental objectives. The pricing strategy influences the return rate negatively, as consumers delay the return of their goods when the purchasing (and repurchasing) price is high. We then compare a wholesale price contract (WPC) and a revenue sharing contract (RSC) mechanism as both have interesting pricing policy implications. Our result shows that firms coordinate the CLSC through a (WPC) when the sharing parameter is too low while the negative effect of pricing on returns is too severe. In that case, the low sharing parameter deters the manufacturer to accept any sharing agreements. Further, firms coordinate the CLSC when the sharing parameter is medium independent of the negative impact of pricing on returns. When the sharing parameter is too high the retailer never opts for an RSC. We find that the magnitude of pricing effect on returns determines the contract to be adopted: For certain sharing parameter, firms prefer an RSC when the price effect on return is low and a WPC when this effect is high. In all other cases, rms do not have a consensus on the contract to be adopted and coordination is then not achieved.

Journal of Economic Literature Classification: L1, M11, D24.
\end{abstract}

Keywords: Closed-Loop Supply Chain, Dynamic return rate, Coordination, Wholesale price contract, Revenue sharing contract. 


\section{Introduction}

A closed loop supply chain (CLSC) integrates forward and backward flows into a unique system, including product acquisition, reverse logistics, points of use and disposal, testing, sorting, refurbishing, recovery, recycling, re-marketing, and re-selling. These activities have to be integrated in the a classical forward system (Guide and Van Wassenhove (2009), Fleischmann et al. (2001)). The recent trend of closed-loop supply chain (CLSC) has highlighted three main aspects that have the merit to be investigated in such a framework: 1. the return rate is a dynamic phenomenon and should be evaluated accordingly (De Giovanni and Zaccour, 2014) 2. consumers return end-of-life/end-of-use products according to the purchasing price (Genc and De Giovanni, 2017); 3. firms partnering in a CLSC always look to the best contracts to be implemented so as to achieve higher economic and environmental performance.

Firms look for the implementation of a CLSC since the returns have some residual value that contributes to the margins. For instance, producing by means of virgin material is always more expensive than producing by means of returned products. Savings vary according to the industry. In the car engines industry, Volkswagen can save up to $70 \%$ of costs (Volkswagen, 2011). Kodak saves $40-60 \%$ of production costs because it manufacturers by means of returned cameras rather than using raw material (Savaskan et al., 2004). Fleischmann et al. (2002) reported that remanufacturing costs at IBM are much lower than those associated with buying new parts, sometimes as much as $80 \%$ lower. Duracell saves $40 \%$ of the production costs when producing by means of returned batteries (De Giovanni, 2017). Xerox saves 40-65\% of its manufacturing costs by reusing parts, components and materials from returned products (Savaskan et al., 2004). Remanufactured cartridges cost $30 \%$ to $60 \%$ less on a per- copy basis than non-remanufactured cartridges. TriNet has been purchasing remanufactured toner cartridges, saving $25 \%$ to $60 \%$ in costs over the price of new cartridges within five years (www.stopwaste.com). Interface, Inc., is the world's largest provider of commercial carpet tile. To create efficiency in the CLSC, the company has decided to lease carpets instead of selling them; the ownership of off-lease products provides Interface motivations to close the loop and recover the residual value of these products (Agrawal and Tokay 2010). Dell saves 30\% of the production cost when recycling their returns (De Giovanni and Ramani, 2018). Manufacturers have high economic interests for performing the backward logistics activities and closing the loop, because the residual value that returns carried out positively contributes to their profits.

Our first contribution is in line with the investigation of the green activity program (GAP) strategy as well as the selling price on returns. The latter represents a novelty with respect to the earlier mentioned literature. The rationale behind this approach is that when the selling price is high, consumers should make considerable sacrifices to purchase it. Therefore, they delay the product return to exploit the good as much 
as they can. This intuition fulfills a research gap in the literature of dynamic games, in which the relationship between returns and pricing has been mainly disregarded. Rather, the static literature reports the negative relationships between pricing and returns. For example, De Giovanni and Zaccour (2018) show that a decision maker can be confronted with sophisticated consumers. When introducing a new product in the market, consumers decide whether to return their product and purchase the updated one according to the price: The higher the price of a product, the lower the return rate, because the lower is the consumers' willingness to return their product and purchase a new one. This model is based on two cases, one from the automotive industry and one from the high-tech industry. The findings obtained apply in both cases; therefore, the results in De Giovanni and Zaccour (2018) corroborate our assumptions on the relationship between pricing and returns. Finally, other research like Zhou et al. (2017) and Miao et al. (2017) model a trade-off between pricing and returns.

Following the early intuitions, we consider a benchmark CLSC setup where the manufacturer optimally sets the green efforts and the retailer sets the selling price in a dynamic framework. Notice that, the retailer participates in determining the return rate through the pricing strategy, but she does not really exploit its potentiality. Then, we contrast the results of the benchmark (WPC) game with an RSC game in which the retailer transfers a share of her revenues to the manufacturer while paying no wholesale price. The manufacturer can find an RSC very interesting to increase the return rate and enjoy the returns' residual value even more. Further, the manufacturer seeks to exploit the property of the RSC that leads to a price reduction (Cachon and Lariviere, 2005).

To recapitulate, we wish to answer the following research questions:

- how do firms in a CLSC set their pricing and GAP strategies when the return rate depends on both pricing and green efforts?

- how do firms' strategies and profits change when moving from a WPC to an RSC setting?

- how do returns change when moving from one setting to another?

Our findings demonstrate that the manufacturer prefers an RSC when more investments in green efforts under an RSC are needed. At the same time, the CLSC performs higher returns as the green efforts have a dominant effect on pricing. When fixing the sharing parameter at a high level, the manufacturer always prefers the RSC. When the sharing parameter is low, its preferences are fully dependent on the pricing effect on returns. When the latter is low, the manufacturer opts for an RSC; otherwise, he will opt for a WPC. The retailer never prefers an RSC when the sharing parameter is too high, because she transfers too much 
revenues to the manufacturer. In contrast, when the sharing parameter is too low, the retailer's preferences mainly depend on the pricing effect on returns. When this is too severe, she will opt for a WPC because the low sharing parameter is not sufficient to properly involve the manufacturer to accept an RSC. Finally, we identify two Pareto-improving regions in which firms reach coordination in CLSC. The first is represented by a low sharing parameter and a high negative effect of pricing on returns, according to which coordination is reached through a WPC. The second region is represented by the case in which the sharing parameter is medium, which calls for the adoption of an RSC, independent of the negative effect of pricing on returns. For certain sharing parameter values, the negative effect of pricing on returns determines the contract to be adopted: An RSC, when this effect is low, and a WPC when this effect is high. In all other cases, the firms have divergent contractual preferences and therefore coordination is never reached.

The remaining of the paper is structure as follows. Section 2 proposes the dynamic games to be analyzed. Section 3 proposes the solutions for all models, while Section 4 compares the games' outcomes. Section 5 briefly concludes.

\begin{tabular}{|l|l|}
\hline Notation & Description \\
\hline$M, R$ & Manufacturer, Retailer \\
\hline$t$ & Time \\
\hline$p(t)$ & Wholesale price at time $t$ \\
\hline$A(t)$ & Price at time $t$ \\
\hline$\phi$ & Green activity programs at time $t$ \\
\hline$\pi_{M}, \pi_{R}$ & Revenue sharing parameter \\
\hline$\Delta$ & Returns' residual value \\
\hline$r(t)$ & Return rate \\
\hline$k$ & Green activity programs sensitivity to $r$ \\
\hline$\lambda$ & Price programs sensitivity to $r$ \\
\hline$\alpha$ & Market potential \\
\hline$\beta$ & Consumers sensitivity to price \\
\hline$D$ & Demand \\
\hline$\rho$ & Discount factor \\
\hline
\end{tabular}

Table 1. Notations 


\section{Dynamic games}

All notations that we use in this paper are listed in Table 1. A Closed-loop Supply Chain (CLSC) is composed of one Manufacturer, firm $M$, and one Retailer, firm $R$. Both firms are involved in the management of both the forward and the reverse flows. $M$ decides the green activity programs to be carried out, namely $A(t)$, which includes, among others, investments in green technologies, green process innovation, green advertising, green marketing and reverse logistics. $R$ is a pure seller that purchases goods from $M$ at a wholesale price, $\omega(t)$, and sets the optimal selling price, $p(t)$, according to the WPC. $R$ 's marginal profits, are the given by $\pi_{R}=p(t)-\omega(t)$, while $M$ 's marginal profits are given by $\pi_{M}=\omega(t)+\Delta r(t) . \Delta r(t)$ represents the marginal benefit that $M$ receives when collecting used-product from the market. We assume that $\Delta>0$ otherwise $M$ would not have any interest in collecting used products. Thus, $\Delta$ is the per-return residual value while $r(t)$ is the fraction of sold goods that will be returned. $r(t)$ is a dynamic stock that takes the form:

$$
\dot{r}(t)=k A(t)-\lambda p(t) \sqrt{r(t)}-\delta r(t)
$$

such that $r(t) \in(0,1)$. When $r(t)=0, M$ does not enjoy any return, while when $r(t)=1$ all consumers return their good when it reaches the end-of-use/life stage. De Giovanni and Zaccour (2013) and De Giovanni et al. (2016) have investigated the return rate as a dynamic equation that mainly evolves according to the GAP investments. Hereby, we introduce also the price effect in the state dynamics following the idea that when consumers pay a high price for their goods, they attempt to substitute it very late over time. First, they try to enjoy the product for as long as they can after the economic sacrifice requested to purchase it. Second, consumers will eventually need to purchase a new product to continue satisfying their needs; therefore, they will again face an important sacrifice when purchasing the product again. According to Eq. (1), firms can make consumers aware of their green investments and sensibilize consumers in returning their products according to the parameter $k>0$, which exemplifies the return rate changes according to the GAP investments that $M$ affords. Further, consumers can delay their return because of the pricing strategy according to the parameter $\lambda>0$, which describes the return rate changes according to $R$ 's pricing strategy. Finally, there is a natural decay rate $\delta$ affecting the return rate state, as consumers forget to send back their end-of-use/life products if they are not exposed to the GAP efforts.

Both the returns and the pricing strategies have an effect on the demand, which can be described as follows:

$$
D(r(t), p(t))=\alpha \sqrt{r(t)}-\beta p(t)
$$


where $\alpha$ is the market potential and explains the amount of consumers exposed to the product and $\beta>0$ is the consumers' sensitivity to price. Interestingly, $M$ 's gives a positive contribution to both the state and the demand, while the opposite applies for R's strategy. We model the GAP efforts strategy using a classical quadratic cost function, e.g., $C(A(t))=\frac{[A(t)]^{2}}{2}$.

Following the same assumptions as in De Giovanni (2017), we start our analysis from a Wholesale Price Contract (WPC) scenario, in which $M$ sells some goods to $R$ at $\omega$ while $R$ sets $p(t)>\omega$ causing an issue of double marginalization. We will use the superscript $\mathcal{W}$ to refer to the WPC game. Accordingly, the firms' profit functions take the following forms:

$$
\begin{aligned}
& J_{M}^{\mathcal{W}}=\max _{\omega^{\mathcal{W}}(t), A^{\mathcal{W}}(t)} \int_{0}^{+\infty} e^{-\rho t}\left[\left(\alpha \sqrt{r^{\mathcal{W}}(t)}-\beta p^{\mathcal{W}}(t)\right)\left(\omega^{\mathcal{W}}(t)+\Delta \sqrt{r^{\mathcal{W}}(t)}\right)-C^{\mathcal{W}}\left(A^{\mathcal{W}}(t)\right)\right] d t \\
& J_{R}^{\mathcal{W}}=\max _{p^{\mathcal{W}}(t)} \int_{0}^{+\infty} e^{-\rho t}\left[\left(\alpha \sqrt{r^{\mathcal{W}}(t)}-\beta p^{\mathcal{W}}(t)\right)\left(p^{\mathcal{W}}(t)-\omega^{\mathcal{W}}(t)\right)\right] d t
\end{aligned}
$$

where $\rho$ is the discount factor that we assume to be the same for the two firms.

One of the main novelty presented in Eq. (1) is the relationship between the return rate, $r(t)$, and the pricing strategy, $p(t)$. Therefore, we evaluate the effectiveness of an RSC within this framework. M's wholesale price takes null values, i.e., $\omega=0$, while $R$ still sets the selling price. We use the superscript $\mathcal{R}$ to refer to the RSC game. Accordingly, the firms marginal profits are given by $\pi_{M}^{\mathcal{R}}=p^{\mathcal{R}}(t) \phi+\Delta \sqrt{r^{\mathcal{R}}(t)}$ and $\pi_{R}^{\mathcal{R}}=p^{\mathcal{R}}(t)(1-\phi)$, where $\phi$ is the sharing parameter and informs on the firms' negotiation on how revenues are shared. Finally, the firms' profits in the $\mathcal{R}$-game are given by:

$$
\begin{aligned}
J_{M}^{\mathcal{R}} & =\max _{A^{\mathcal{R}}(t)} \int_{0}^{+\infty} e^{-\rho t}\left[\left(\alpha \sqrt{r^{\mathcal{R}}(t)}-\beta p^{\mathcal{R}}(t)\right)\left(p^{\mathcal{R}}(t) \phi+\Delta \sqrt{r^{\mathcal{R}}(t)}\right)-C^{\mathcal{R}}\left(A^{\mathcal{R}}(t)\right)\right] d t \\
J_{R}^{\mathcal{R}} & =\max _{p^{\mathcal{R}}(t)} \int_{0}^{+\infty} e^{-\rho t}\left[\left(\alpha \sqrt{r^{\mathcal{R}}(t)}-\beta p^{\mathcal{R}}(t)\right) p^{\mathcal{R}}(t)(1-\phi)\right] d t
\end{aligned}
$$

Both the $\mathcal{W}$-game and the $\mathcal{R}$-game are played á la Stackelberg. We resolve the game by assuming that the players use a stationary feedback strategy, which is standard in differential games over the infinite time horizon (Dockner et al., 2000). When modeling CLSCs, one would resolve the game using an open-loop strategy due to the complex relationships between controls and states (Genc and De Giovanni, 2018). Although we cannot obtain an analytical solution by using feedback strategies, we can derive a time-consistent equilibrium. Moreover, the value of the information obtained in the feedback strategy is much more appropriate from a managerial perspective in both channel and supply chain management studies. 


\section{Equilibria}

In this section, we present the solutions for the dynamic games earlier introduced. In both cases, we solve the games á la Stackelberg, where $M$ is the leader. As for conventional solutions in dynamic games with infinite time horizon, all strategies and value functions are written as a function of the state.

\subsection{A dynamic CLSC using a wholesale price contract - $\mathcal{W}$-Scenario}

In this section, we present the solution of the $W$-game. Hereby, $M$ decides the green efforts, $A(t)$, along with the wholesale price, $\omega(t)$, when selling the products to $R$. The latter charges a selling price, $p(t)$, such that $p(t)>\omega(t) . M$ announces that the chain uses a WPC to regulate the financial flow; $R$ considers this announcement and decides the optimal selling price, $p(t)$; $M$ takes $p(t)$ into consideration and optimally sets its controls. The firms' strategies and profits are summarized in the following proposition.

Proposition 1 The equilibrium strategies in the $\mathcal{W}$-Scenario are given by:

$$
\begin{aligned}
\omega^{\mathcal{W} *} & =\frac{\left(\lambda L_{1}^{\mathcal{W}}-\left(\Delta \beta+\lambda B_{1}^{\mathcal{W}}\right)\right)}{\beta} \sqrt{r^{\mathcal{W}}} \\
p^{\mathcal{W} *} & =\frac{\left(\alpha-\Delta \beta-\lambda B_{1}^{\mathcal{W}}\right)}{2 \beta} \sqrt{r^{\mathcal{W}}} \\
A^{\mathcal{W} *} & =\frac{k B_{1}^{\mathcal{W}}}{\mu}
\end{aligned}
$$

where the pairs $\left(B_{i}^{\mathcal{W}}, L_{i}^{\mathcal{W}}\right)$ with $i=1,2$ are the coefficients of the value functions $V_{M}^{\mathcal{W}}\left(r^{\mathcal{W}}\right)$ and $V_{R}^{\mathcal{W}}\left(r^{\mathcal{W}}\right)$, which are given by:

$$
\begin{aligned}
& V_{M}^{\mathcal{W} *}=B_{1}^{\mathcal{W}} r^{\mathcal{W}}+B_{2}^{\mathcal{W}} \\
& V_{R}^{\mathcal{W} *}=L_{1}^{\mathcal{W}} r^{\mathcal{W}}+L_{2}^{\mathcal{W}}
\end{aligned}
$$

These value functions describe the optimal profits along the optimal return rate trajectory, $r^{\mathcal{W}}(t)$. The optimal time-path of the return rate reads as follows:

$$
r^{\mathcal{W}}(t)=\left(r_{0}-r_{S S}^{\mathcal{W}}\right) e^{-t\left[\mu\left(\left(\alpha-\Delta \beta-\lambda B_{1}^{\mathcal{W}}\right) \lambda+2 \beta \delta\right)\right]}+r_{S S}^{\mathcal{W}}
$$

where $r_{S S}^{\mathcal{W}}$ is the steady-state return rate and is given by:

$$
r_{S S}^{\mathcal{W}}=\frac{2 \beta B_{1}^{\mathcal{W}} k^{2}}{\mu\left(\left(\alpha-\Delta \beta-\lambda B_{1}^{\mathcal{W}}\right) \lambda+2 \beta \delta\right)}
$$


Proof. See the Appendix.

From the equilibria, one can see that value functions are linear in the state variable $r^{\mathcal{W}}$, although the state equation contains the square root of $r$. The reason is that the present games is a special case of the framework dating back to Sethi (1983) and also discussed in Sethi and Thompson (1981) and Dockner et al. (2000).

From the Appendix, we can see that $B_{1}^{\mathcal{W}}$ has two roots, specifically:

$$
B_{1}^{\mathcal{W}}=\frac{\left((\alpha-\Delta \beta) \lambda+2 \beta(\delta+\rho) \pm 2 \sqrt{\beta(\delta+\rho)((\alpha-\Delta \beta) \lambda+\beta(\delta+\rho))-\Delta \alpha \beta \lambda^{2}}\right)}{\lambda^{2}}
$$

Both of them are positive as the price, $p$, is always larger than the returns' value, $\Delta$. Thus, $\alpha-\Delta \beta>0$. We can take the negative root out. This guarantees that $A_{S S}^{\mathcal{\mathcal { W } _ { * }}}>0$. Notice that the green efforts strategies are state independent, meaning that $M$ sets the green efforts while disregarding the value of the stock. Intuitively, the impact of green efforts efficiency, exemplified by $k$ in the state and $\mu$ in the cost function, suggests that when $M$ should increase or decrease these efforts. From the Appendix, one can see that $L_{1}^{\mathcal{W}}$ is given as follows:

$$
L_{1}^{\mathcal{W}}=\frac{\mu\left(\alpha+\Delta \beta+\lambda B_{1}^{\mathcal{W}}\right)^{2}}{4 \mu(\alpha \lambda-\beta(\delta+\rho))}
$$

This guarantees that $p^{\mathcal{W} *}>\omega^{\mathcal{W} *}$ always holds at the steady-state, as $p^{\mathcal{W} *}-\omega^{\mathcal{W} *}=\frac{\left(\alpha+\Delta \beta+\lambda B_{1}^{\mathcal{W}}-2 \lambda L_{1}^{\mathcal{W}}\right)}{2 \beta}>0$. Both the price and wholesale price strategies depend on the return rate. Therefore, when the CLSC performs the return rate, firms know that the portfolio of consumers increases, generating more market potential. In this case, increasing the prices will not be detrimental for sales. The latter takes the following form:

$$
D^{\mathcal{W}}=\frac{\left(\alpha+\left(2 \Delta \beta+\lambda B_{1}^{\mathcal{W}}\right)\right)}{2} \sqrt{r^{\mathcal{W}}}
$$

Interestingly, we can see that there is a trade-off between environmental performance, given by $r^{\mathcal{W}}$, and the double marginalization effect, $p^{\mathcal{W} *}-\omega^{\mathcal{W} *}$, as increasing the return rate is detrimental for consumers, who are subject to higher prices. Since $B_{1}^{\mathcal{W}}>0$ and $L_{1}^{\mathcal{W}}>0$, both firms have a certain convenience in increasing the returns. This result has a direct and positive effect on their profits, given that $\frac{\partial V_{M}^{\mathcal{W} *}}{\partial r^{\mathcal{W}}}=B_{1}^{\mathcal{W}}>0$ and $\frac{\partial V_{R}^{\mathcal{W} *}}{\partial r^{\mathcal{W}}}=L_{1}^{\mathcal{W}}>0$. From the Appendix, we can also see that

$B_{2}^{\mathcal{W}}$ and $L_{2}^{\mathcal{W}}$ are always positive, given that:

$$
B_{2}^{\mathcal{W}}=\frac{B_{1}^{2} k^{2}}{2 \mu \rho} \text { and } L_{2}^{\mathcal{W}}=\frac{L_{1}^{\mathcal{R}} B_{1}^{\mathcal{W}} k^{2}}{\mu \rho}
$$


Consequently, the CLSC business is economically interesting for both firms even if the return rate is negligible. Finally, $r_{S S}^{\mathcal{W}}$ is globally asymptotically stable because $\left(\alpha-\Delta \beta-\lambda B_{1}^{\mathcal{R}}\right) \lambda+2 \beta \delta>0$. Accordingly,

the return rate is positive at the steady-state. Note that $\lim _{\lambda \rightarrow 0} r_{S S}^{\mathcal{W}}=\frac{B_{1}^{\mathcal{W}} k^{2}}{\mu \delta}$, thus highlighting the considerable impact of pricing on the returns. These circumstances create the basis to evaluate an alternative price-based mechanism to mitigate this negative effect.

\subsection{A dynamic CLSC using a revenue sharing contract - $\mathcal{R}$-Scenario}

Next we solve the $\mathcal{R}$-game, in which firms use the revenue sharing contract to coordinate their financial flows. The WPC earlier described leaves the issue of double marginalization effect, as $p(t)>\omega(t)$ always holds. This can be very detrimental for sales, as the double marginalization leads to lower sales. In addition, since pricing is also influencing negatively the returns (e.g., Eq. 1), there is a further interest to look into a mechanism to mitigate the negative effects on the return rate. In this setting, $M$ only decides the green efforts, $A(t)$, while $R$ charges a selling price, $p(t)$ without being subject to the constraint $p(t)>\omega(t)$. In addition, firms share the revenues generated by the business through the sharing parameter $\phi \in(0,1)$. M announces that the chain uses an RSC to regulate the financial flows; $R$ considers this announcement and decides the optimal selling price, $p(t) ; M$ takes $p(t)$ into consideration and optimally sets $A(t)$. The firms' strategies and profits are summarized in the following proposition.

Proposition 2 The equilibrium strategies in the $\mathcal{R}$-Scenario are given by:

$$
\begin{aligned}
p^{\mathcal{R} *} & =\frac{\alpha(1-\phi)-\lambda L_{1}^{\mathcal{R}}}{2 \beta(1-\phi)} \sqrt{r} \\
A^{\mathcal{R} *} & =\frac{k B_{1}^{\mathcal{R}}}{\mu}
\end{aligned}
$$

where the pairs $\left(B_{i}^{\mathcal{R}}, L_{i}^{\mathcal{R}}\right)$ are the coefficients of the value functions $V_{M}^{\mathcal{R}}\left(r^{\mathcal{R}}\right)$ and $V_{R}^{\mathcal{R}}\left(r^{\mathcal{R}}\right)$, which are given by:

$$
\begin{aligned}
V_{M}^{\mathcal{R}} & =B_{1}^{\mathcal{R}} r^{\mathcal{R}}+B_{2}^{\mathcal{R}} \\
V_{R}^{\mathcal{R}} & =L_{1}^{\mathcal{R}} r^{\mathcal{R}}+L_{2}^{\mathcal{R}}
\end{aligned}
$$

These value functions describe the optimal profits along the optimal return rate trajectory, $r^{\mathcal{R}}(t)$. The optimal time-path of the return rate reads as follows:

$$
r^{\mathcal{R}}(t)=\left(r_{0}-r_{S S}^{\mathcal{R}}\right) e^{-t\left[\mu\left((\alpha \lambda+2 \beta \delta)(1-\phi)-\lambda^{2} L_{1}\right)\right]}+r_{S S}^{\mathcal{R}}
$$


where $r_{S S}^{\mathcal{R}}$ is the steady-state return rate and is given by:

$$
r_{S S}^{\mathcal{R}}=\frac{2(1-\phi) \beta B_{1} k^{2}}{\mu\left((\alpha \lambda+2 \beta \delta)(1-\phi)-\lambda^{2} L_{1}^{\mathcal{R}}\right)}
$$

Proof. See the Appendix.

From the Appendix, we can see that $B_{1}^{\mathcal{R}}$ has one unique solution, specifically:

$$
B_{1}^{\mathcal{R}}=\frac{\mu\left(\lambda L_{1}^{\mathcal{R}}+\alpha(1-\phi)\right)\left((2 \Delta \beta+\alpha \phi)(1-\phi)-\lambda \phi L_{1}^{\mathcal{R}}\right)}{2 \mu(1-\phi)\left((\lambda \alpha+2 \beta(\delta+\rho))(1-\phi)-\lambda^{2} L_{1}^{\mathcal{R}}\right)} .
$$

This is a positive expression, which guarantees that $A_{S S}^{\mathcal{R} *}>0$. Note that, even in the revenue sharing setting, the green efforts strategies are state independent, meaning that $M$ always disregards the value of the stock when setting the green efforts, independent of the contract the CLSC uses. Further, the green efforts structure is similar to the one derived in the WPC contract, thus we can refer to the previous discussion. From the Appendix, one can see that $L_{1}^{\mathcal{W}}$ has two roots, one of which is positive and one of which is negative. To have positive returns, we take the negative root out, which is given by:

$$
L_{1}^{\mathcal{R}}=\frac{(\alpha \lambda+2 \beta(\delta+\rho))(1-\phi)-2 \sqrt{\beta(1-\phi)^{2}(\delta+\rho)(\alpha \lambda+\beta(\delta+\rho))}}{\lambda^{2}} .
$$

Further, this guarantees that $r^{\mathcal{R}} \in(0,1)$ and $p^{\mathcal{R}}>0$. The price positively depends on the return rate. Therefore, when the CLSC performs the return rate, firms can charge a higher price. Interestingly, when firms enjoy high returns, they also enjoy a higher market potential, which allows them to increase the price to extract more economic value from the market. Also, this result informs researchers on the importance of dynamic elements: the contribution that the state, $r^{\mathcal{R}}(t)$, gives to the firms' profit function is more important than a single strategy, e.g., pricing. Therefore, CLSC is a dynamic phenomenon and should be studied as such. Consequently, the sales under a revenue sharing contract are given by:

$$
D^{\mathcal{R}}=\frac{\alpha(1-\phi)+\lambda L_{1}^{\mathcal{R}}}{2(1-\phi)} \sqrt{r^{\mathcal{R}}}
$$

Since $B_{1}^{\mathcal{R}}>0$ and $L_{1}^{\mathcal{R}}>0$, both firms have a certain convenience in contributing for increasing the returns. This result has a direct and positive effect on their profits, given that $\frac{\partial V_{M}^{\mathcal{R} *}}{\partial r^{\mathcal{R}}}=B_{1}^{\mathcal{R}}>0$ and $\frac{\partial V_{R}^{\mathcal{R} *}}{\partial r^{\mathcal{R}}}=L_{1}^{\mathcal{R}}>0$. From the Appendix, we can also see that $B_{2}^{\mathcal{R}}$ and $L_{2}^{\mathcal{R}}$ are always positive, given that:

$$
B_{2}^{\mathcal{R}}=\frac{B_{1}^{\mathcal{R}^{2}} k^{2}}{2 \mu \rho} \text { and } L_{2}^{\mathcal{R}}=\frac{L_{1}^{\mathcal{R}} B_{1}^{\mathcal{R}} k^{2}}{\mu \rho}
$$


As in the WPC, the CLSC business is economically interesting for both firms even if the return rate is neglegible. Finally, $r_{S S}^{\mathcal{R}}$ is globally asymptotically stable when $\mu\left((\alpha \lambda+2 \beta \delta)(1-\phi)-\lambda^{2} L_{1}^{\mathcal{R}}\right)>0$. Accordingly, the return rate is positive at the steady-state. Note that $\lim _{\lambda \rightarrow>0} r_{S S}^{\mathcal{R}}=\frac{B_{1}^{\mathcal{R}} k^{2}}{\delta \mu}$, thus highlighting the considerable impact of pricing on the returns. Interestingly, when the returns are not affected by pricing, the return rate takes the same structure as in the $\mathcal{W}$-game. We seek now to evaluate the impact of the sharing parameter, $\phi$, on the firms' strategies and profits as well as on the sales.

Numerically, we can see that $\frac{\partial B_{1}^{\mathcal{R}}}{\partial \phi}>0$ and $\frac{\partial L_{1}^{\mathcal{R}}}{\partial \phi}<0$. Figure 1 displays the relationship between the coefficients and the sharing parameter, $\phi^{1}$.

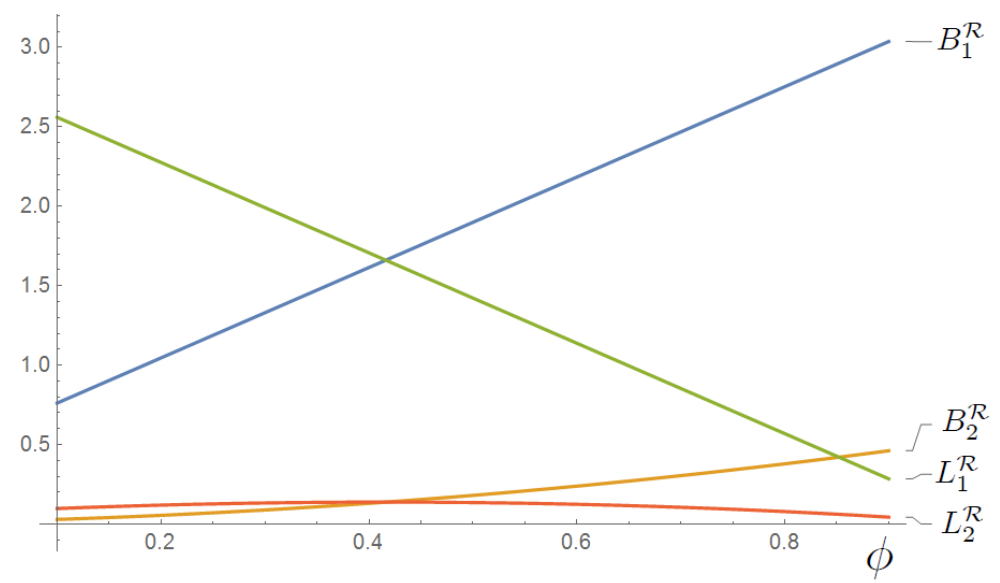

Figure 1. The relationship between the coefficients $B_{i}, L_{i}$ with respect to $\phi$.

Consequently, we can formulate the following corollary:

Corollary 1 The behavior of strategies, demand and profits with respect to the sharing parameter, $\phi$, is as follows: $\frac{\partial A_{S S}^{\mathcal{R}}}{\partial \phi}>0, \frac{\partial r_{S S}^{\mathcal{R}}}{\partial \phi}>0, \frac{\partial p_{S S}^{\mathcal{R}}}{\partial \phi}>0, \frac{\partial D_{S S}^{\mathcal{R}}}{\partial \phi}>0 ; \frac{\partial V_{M_{S S}^{\mathcal{R}}}^{\mathcal{R}}}{\partial \phi}>0$ and $\frac{\partial V_{R_{S S}}^{\mathcal{R}}}{\partial \phi} \geqslant 0$, $\forall \phi \in(0, \bar{\phi}]$.

\section{Proof. See the Appendix.}

The results of Corollary 1 are clearly displayed in Figures 2 and 3.

Accordingly, we can leave the following remarks. The RSC does not provide the usual benefits of decreased price as claimed by Cachon (2003). This is due to the fact that the pricing influences the state variable, which decreases due to the higher price. This implication calls $M$ to invest more in green efforts, $A^{\mathcal{R}}$, given the fact that he is receiving a share: higher share implies higher economic availability, and thus a larger chance

\footnotetext{
${ }^{1}$ We carry out the numerical analysis by setting the parameters at the following values:

$\alpha=2, \beta=0.6, \delta=0.2, \rho=0.1, \lambda=0.2, \mu=1, \Delta=0.2, k=0.5$. Instead, we leave $\phi$ as free.
} 
to increase the green efforts. At the same time, $R$ needs more economic resources now, since a part of her revenues is transferred to $M$. The joint effect of pricing and green efforts translates into higher returns and sales, thus we assist to a positive reaction from the market that returns used goods and purchases new ones. Finally, there is a tough negotiation to be carried out before starting the game on the sharing parameter. While $M$ is always happy to receive a share, $R$ benefits from it til a certain level. In fact, when the sharing parameter is too high, e.g., $\phi>\bar{\phi}, R$ transfers too much revenues to $M$ with the results that her profits decrease. Interestingly, when $\phi=\bar{\phi}$, the firms gain the same profits.

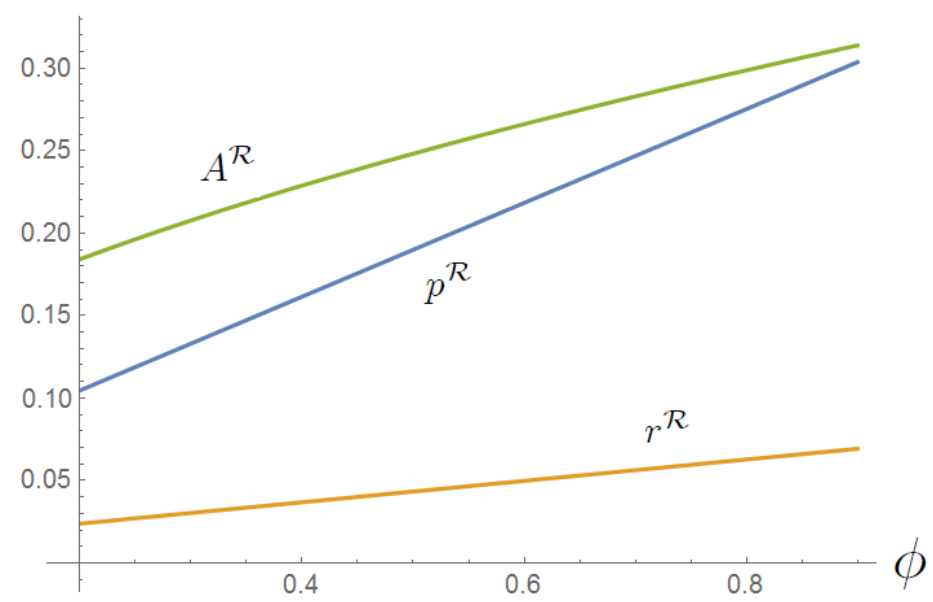

Figure 2. Price, green efforts and returns with respect to $\phi$.

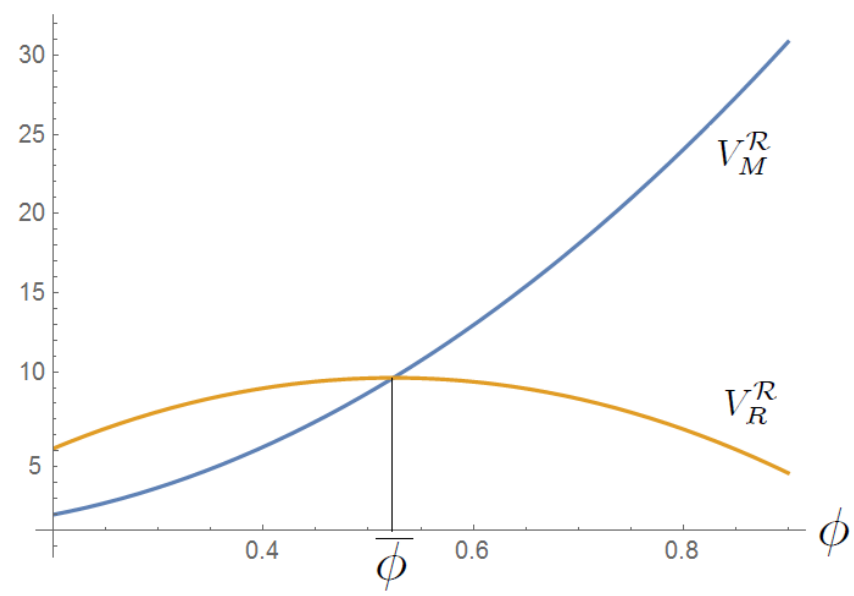

Figure 3. The relationship between the $V_{M}$ snd $V_{R}$ with respect to $\phi$. 


\section{Comparison between games}

In this section we compare the outcomes of both the WPC and the RSC games to answer our initial research questions. We use the same benchmark parameters that have been previously set while we focus in the $(\phi, \lambda)$ - space analysis. The motivations for doing so are twofold. First, the analysis of the sharing parameter will inform on the efficiency of an RSC with respect to a WPC in the exchange of financial flows. Second, the sharing parameter has a direct effect on pricing. Thus, the impact of pricing on the return rate adds new insights in this literature frame.

From Figure 4, it is interesting to see that $M$ adjusts the green efforts according to both the sharing parameter value and the impact of pricing on returns. When these two effects are low, $M$ is not very much interested in the business; instead, he does care about $R$ 's power. Even when he gets a minimal revenue and/or there is no impact of $R$ on the returns. When the sharing parameter is high, $M$ has a large incentive for investing in green efforts. When the share increases, he invests more than in the WPC. We can see that when the pricing strategy becomes very severe (for example when the pricing strategy in revenue sharing is much larger than the pricing strategy in the wholesale price contract) the manufacturer wishes to invest more in green efforts in order to overcome the negative effect of the pricing strategy. Nevertheless, this only happens when the business is interesting for $M$, that is, when the sharing parameter is high. Otherwise, when the sharing parameter is low, $M$ does not invest more in green efforts when a revenue sharing contract is implemented.

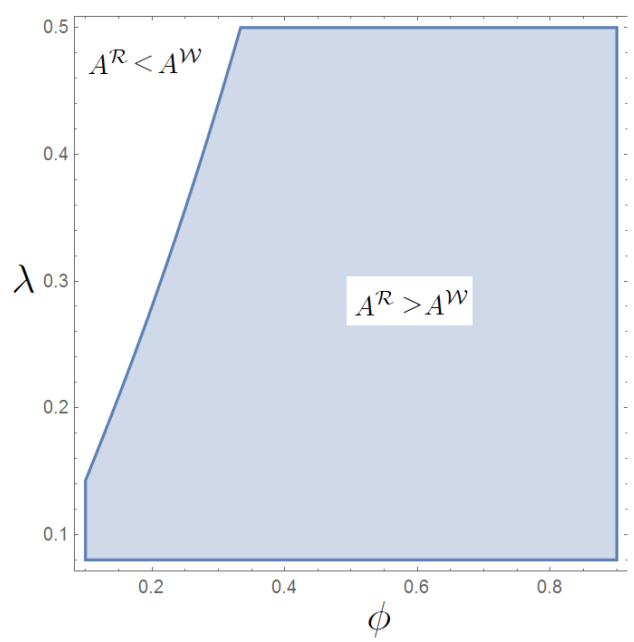

Figure 4. Comparison between green efforts, $A$ 
Figure 5 displays the areas in which $p^{\mathcal{R}}>p^{\mathcal{W}}$. Intuitively, when the sharing parameter is too large, $R$ transfers a considerable amount to $M$; therefore, she needs to set a higher price to make the business profitable. In this case, the CLSC obtains the reverse effect of what we expect from an RSC, that is, the price reduction compared to the WPC setting. We can see that this only happens when the sharing parameter, $\phi$, is sufficiently small. Further, $R$ sets higher prices in RSC when the negative impact of pricing on returns increases. This is a counter-intuitive result as we expect $R$ to lower the price for high values of $\lambda$. In fact, this happens only when $R$ retains a sufficiently large amount of revenues. For example, when the sharing parameter is low, $R$ retains more revenues than $M$, thus she sets an RSC price lower than a WPC price. Nevertheless, when her fraction is low, she needs more economic resources. Then, she opts for charging larger prices while expecting $M$ to invest more in green efforts.

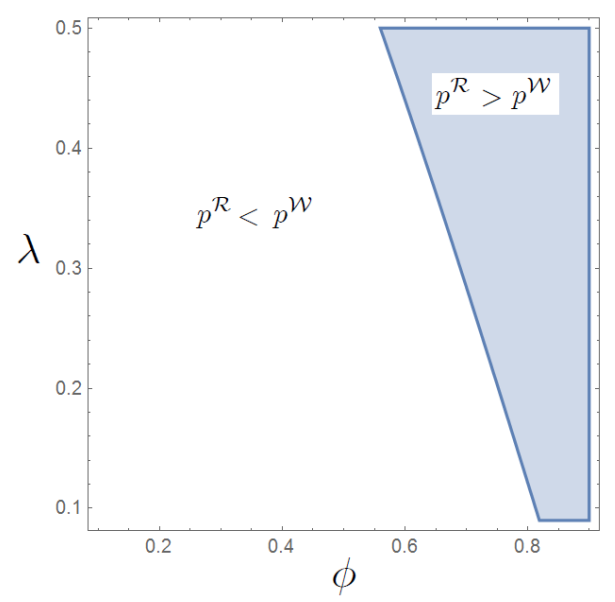

Figure 5. Comparison between pricing strategies

Figure 6 displays the comparison between the return rate at the steady-state in the two proposed games. Here, the returns most likely reflect the green efforts and the pricing strategies. We can see that when the latter becomes more important, the returns decrease more under an RSC. This also happens when the sharing rate is low. Therefore, $M$ does not invest too much in green activity efforts because the business is not appealing, while the opportunities for building an effective reverse flow are minimal. We can see that, in order to enjoy a very efficient CLSC, the sharing parameters should be high while the impact of pricing on returns should be minimal. Supply chains will be very much efficient in managing their own returns if and only if the customers perceive that the pricing doesn't affect the returns. In this sense the CLSC can give an interesting rebate to customers to increase their willingness to return the products and, therefore, have a lower pricing effect on returns (Genc and De Giovanni, 2018). 


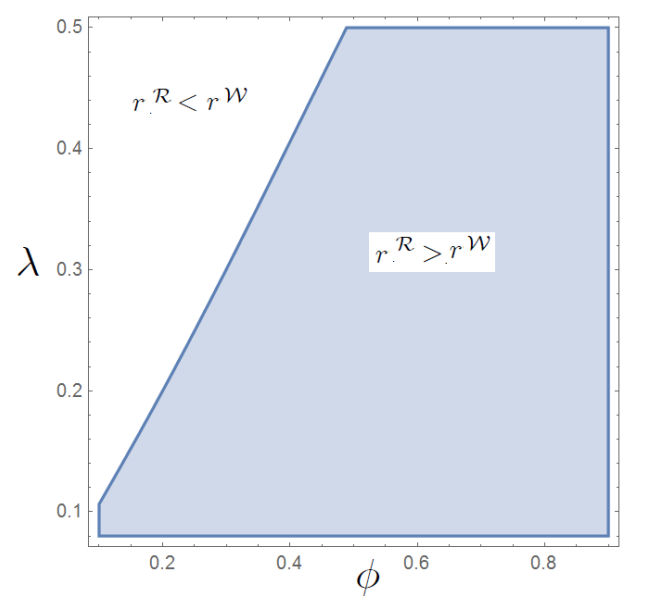

Figure 6. Comparison between return rates, $r$

Figure 7 compares the sales in the two games that we investigate. Accordingly, the adoption of an RSC allows the CLSC to sell more products in the market in most of the cases. In this regard, an RSC is more socially sustainable as more people access to the product. It is interesting to see that this happens also when $p^{\mathcal{R}}>p^{\mathcal{W}}$, thus firms can optimally adjust the green efforts to create a compensation effect when the price is detrimental for both the sales and the returns. Finally, there is only one case in which the sales under an RSC are lower than the sales under a WPC, that is, when $\lambda$ is very high. In this case, the negative effects generated by a pricing strategy are too severe and the green efforts are not sufficiently high.

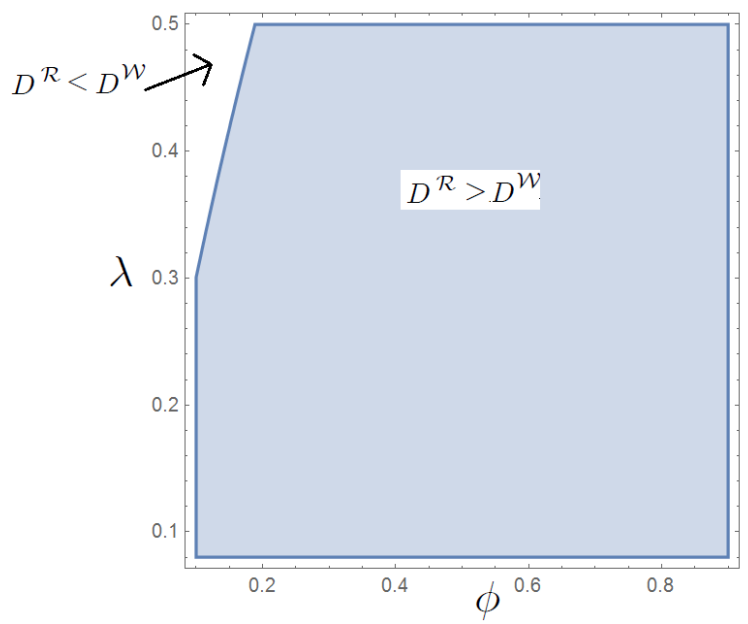

Figure 7. Comparison between sales, $D$

Figure 8 displays the $M$ 's preferences in the selection of the coordination mechanism. This shape is very 
similar to the one in Figure 4 and highlights a clear message. When the sharing parameter is sufficiently large, $M$ always supports the adoption of an RSC. This is very much intuitive as the larger the sharing parameter, the higher the fraction of revenues that goes in his pocket. Interestingly, this finding holds true independent of the negative effect that pricing has on returns. Therefore, under these circumstances, $M$ is always willing to invest more in green efforts to overcome the negative effects generated by pricing, as he gains sufficiently large revenues to be reinvested in green initiatives. Instead, when the sharing parameter is medium vs. low, the convenience of adopting an RSC highly depends on $\lambda$ : when $\lambda$ is low, $M$ always opts for an RSC; when $\lambda$ is high, M's preferences for an RSC decrease according to increasing values for $\lambda$. In the latter case, the negative impact of pricing on returns is too severe, and investing more in green efforts translates in a marginally convenient option.

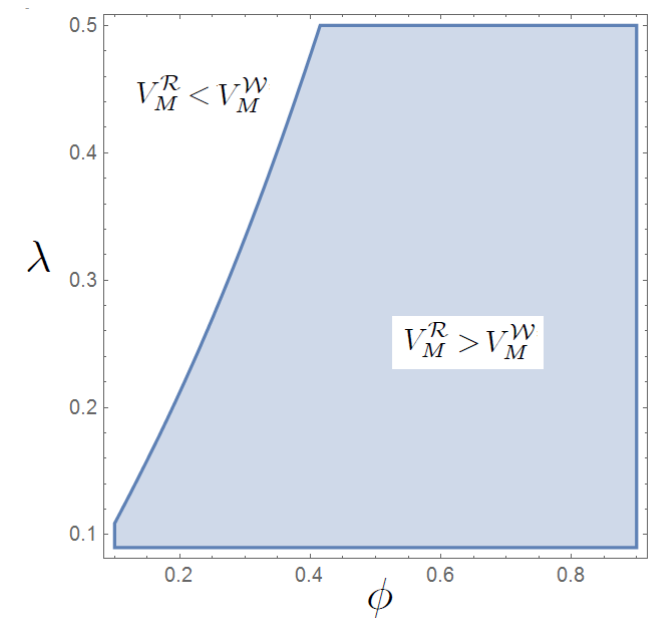

Figure 8. Comparison between $M$ 's profits, $V_{M}$

Figure 9 displays $R$ 's preferences with respect to the coordination mechanism to be adopted. We can mainly identify three regions:

1. The sharing parameter, $\phi$, and the impact of pricing on returns, $\lambda$, are too high and the RSC is a non-feasible (n.f.) option for $R$. Hereby, the revenues transferred to $M$ are too high and her profits become negative. So, when the sharing parameter is too high, $R$ will never be interested in the business if $\lambda$ is also high. In contrast, when $\lambda$ is low, $R$ profits are positive and she opts for the adoption of an RSC. Therefore, the impact of pricing on returns plays a key role in determining whether $R$ finds an RSC convenient for coordinating the CLSC. 
2. The sharing parameter, $\phi$, is very low and the impact of pricing on returns, $\lambda$, is high. In this region, $R$ should be very much happy to coordinate the CLSC through an RSC because her fraction of revenues is the largest. Nevertheless, the negative impact of pricing on returns has a very detrimental effect on this preference; thus, $R$ opts for a WPC when the effect of pricing on returns is too severe. Again, it is interesting to see that the negative effect that pricing exerts on returns by delaying the moment in which consumers return the product has a considerable weight on the $R$ 's preferences.

3. In all cases that are not contemplated in 1 and $2, R$ always opt for an RSC, independent of $\lambda$.

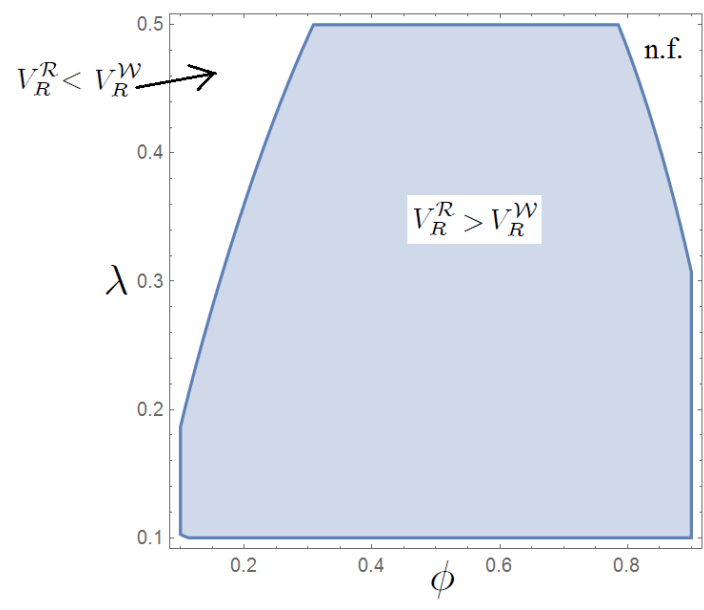

Figure 9. Comparison between $R$ 's profits, $V_{R}$

Finally, Figure 10 informs on the regions in which a Pareto-improving situation is realized. Four regions are identified.

1. The blue region highlights that both firms have a preference for an RSC. As mentioned, the role of $\lambda$ is vital to determine whether the adoption of an RSC leads to a Pareto-improving situation.

2. The orange region indicates that both firms have a preference for a WPC. Hereby, the sharing parameter is too low to engage $M$ in investing in green efforts such that the whole CLSC gets benefits. Also, the impact of pricing on returns is too detrimental, thus backward activities simply become less important.

3. Firms have divergent preferences (d.f.), specifically: $R$ opts for an RSC while $M$ would prefer a WPC. The sharing parameter seems to be too low for $M$ to convince him in implementing an RSC.

4. There exists a non-feasible (n.f.) region in which $R$ would opt for a WPC while $M$ would select a RSC. The latter is not feasible for $R$, thus firms have divergent preferences.

We would highlight the role that the parameter $\lambda$ can have in moving firms' preferences for a contract to another. Let's take for example the case of $\phi=0.2$. When $\lambda$ is low, firms reach coordination through an 
RSC: although $M$ receives a low share, the marginal impact of pricing does not hurt the return rate. Thus, he does not need to invest too much in green efforts to perform the return rate. When $\lambda$ is medium, firms have divergent preferences and coordination is never reached. In this case, $M$ gains through the $R$ 's share of revenues is too low to induce higher investments in green efforts. When $\lambda$ is very high, both firms prefer the adoption of a WPC; $M$ will invest the minimum efforts in the return rate and, consequently, backward activities and closing the loop become negligible targets.

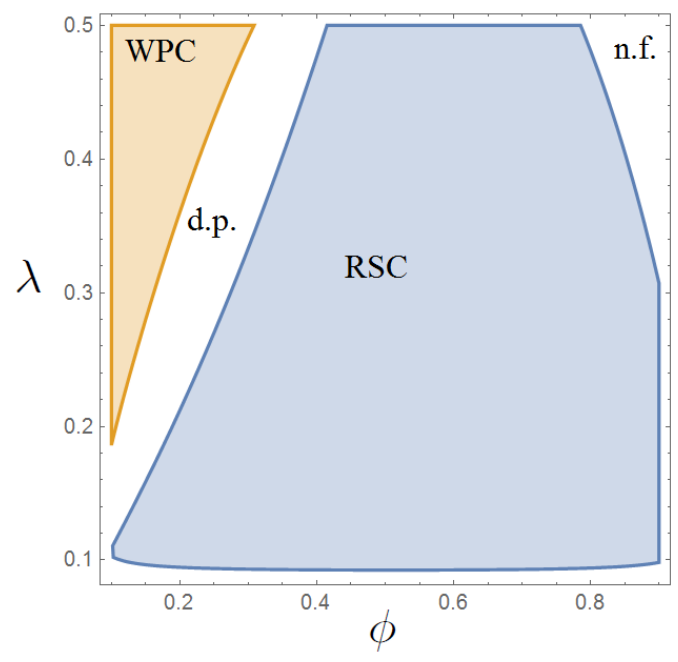

Figure 10. Pareto-improving region

\section{Conclusions}

This paper studies a dynamic CLSC that is involved in managing both the backward and the forward flows of goods. The firms being part of the CLSC, namely, manufacturer and retailer, coordinate their financial flows by choosing between a wholesale price contract (WPC) or a revenue sharing contract (RSC). The manufacturer is the chain leader and fully benefits from the returns' residual value. Nevertheless, the return rate, which consists of the fraction of past sold products that comes back to the manufacturer's plants to be either reused or recycled, is negatively influenced by the pricing strategy. The latter is set by the retailer. This is the main contribution to the literature of dynamic games in CLSC, which focuses on increasing the return rate by some green efforts, generally set by a manufacturer. In addition to that, we also model a return rate that is negatively influenced by the retailer's pricing. The motivations for this assumption lie on the relationship between consumers' willingness to return a product and the product value. When consumers pay high prices for purchasing goods, they will be more parsimonious in returning them. In particular, they will postpone the return with the purpose of exploiting the returns residual value as much as possible. According 
to this assumption, we first model a WPC game, in which the manufacturer sets the green efforts along with the wholesale price, while the retailer sets the pricing strategy. Later we model an RSC game, in which the manufacturer does not set the wholesale price anymore, while the firms share the revenues generated within the CLSC according to an exogenous sharing parameter. The motivations for contrasting a wholesale price to a revenue sharing contract lies on the fact that the implementation of an RSC generally leads to a decrease of the selling price, with the purpose of increasing the demand, thus generating more revenues. The reduction of the selling price in the CLSC can be a driver to limit the negative effect of pricing on the return rate.

Our results show that the manufacturer invests more in a CLSC using an RSC when the sharing parameter is sufficiently high. In fact, this investment fully depends on the amount of money that the manufacturer receives. In such cases, the negative effect of pricing on returns is not a problem. Nevertheless, when the latter effect is severe, the manufacturer does not invest more in an RSC when the sharing parameter is low. In that case, his revenues will be too low to allow the green efforts to compensate for the negative effect of pricing. The CLSC enjoys the positive effect of price reduction when the sharing parameter is not too high. Therefore, there is a need to negotiate the sharing parameter before starting the game in order to get the desired effect. In fact, when the sharing parameter is too high, the retailer needs to charge a higher price than the WPC price because she needs more economic resources to consider the business as interesting. The two strategies (green efforts and pricing) contribute to the return rate with opposite sign. Nevertheless, we find that the return rate shape follows the green efforts shape, which seems to have a dominant effect with respect to the pricing strategy. Thus, any time the manufacturer invests more due to the implementation of an RSC, the returns turn out to be higher than the WPC case. Instead, the consumers enjoy an efficient combination of pricing and green efforts when an RSC is adopted. Thus, the RSC is socially sustainable in most of the cases. The manufacturer prefers the adoption of an RSC any time he invests more in green efforts under an RSC while the CLSC performs higher returns. The sharing parameter plays a key role. It is sufficient to have a high sharing parameter to make the manufacturer always prefers the RSC. When the sharing parameter is low, his preferences will be fully dictated by the influence of pricing on returns. When the latter effect is low, the manufacturer prefers an RSC; otherwise, he will opt for a WPC. On her side, the retailer never opts for an RSC when the sharing parameter is too high, because she transfers a fraction that is not economically viable for her. In contrast, when the sharing parameter is too low, the retailer's preferences are mainly driven by the effect of pricing on returns. When this is too severe, she will opt for a WPC because the sharing parameter will be too low to convince the manufacturer to accept the deal. Finally, we identify two Pareto-improving regions, which represent the areas in which firms reach coordination in CLSC. The first is represented by a low sharing parameter and a high negative effect of pricing on returns. In that case, both firms prefer a WPC. The second area is represented by the case in which the sharing parameter is medium. 
In that case, both firms will prefer an RSC, independent of the negative effect of pricing on returns. For certain sharing parameter values, the target of coordination fully depends on the negative effect of pricing on returns: when this is low, an RSC allows firms to reach coordination; when this effect is high, a WPC allows firms to achieve coordination. In all other cases, the firms have divergent contractual preferences and coordination is never reached.

This research is not free of limitations, which are listed here to inspire future research in this direction. We assume that the CLSC does not experience any competitive effect within each tier. Introducing competition in the manufacturer and/or at the retailer levels will probably change some of our results. We model coordination while considering that the retailer never benefits of returns, as consumers directly send back products to the manufacturer. Having the retailer involved in the collection process will need a further reflection on the coordination mechanisms to be adopted. We assume that the returns' residual value is exogenous and fixed, while it most likely depend on how consumers used the product during the residence time. Therefore, the consumers' behavior also matters in the determination of the goods' residual value. We have modeled a negative impact of pricing on returns. Nevertheless, additional research can be carried out to show the positive effect of pricing on a state variable like green goodwill and, consequently, modeling the return rate as a function of the stock. This would definitely lead to completely different results as pricing would positively influence the returns. This is an ongoing research direction that the authors are exploring.

\section{Appendix}

Proof. of Proposition 1. We search for a pair of bounded and continuously differentiable value functions $V_{M}^{\mathcal{W}}\left(r^{\mathcal{W}}\right)$ and $V_{R}^{\mathcal{W}}\left(r^{\mathcal{W}}\right)$ for which a unique solution for $r^{\mathcal{W}}(t)$ does exist, and the HJB equations:

$$
\begin{aligned}
& \rho V_{M}^{\mathcal{W}}=\left(\alpha \sqrt{r^{\mathcal{W}}}-\beta p^{\mathcal{W}}\right)\left(\omega^{\mathcal{W}}+\Delta \sqrt{r^{\mathcal{W}}}\right)-\frac{\mu\left(A^{\mathcal{W}}\right)^{2}}{2}+V_{M}^{\mathcal{W}}\left(k A^{\mathcal{W}}-\lambda p^{\left.\mathcal{W} \sqrt{r^{\mathcal{W}}}-\delta r^{\mathcal{W}}\right)}\right. \\
& \rho V_{R}^{\mathcal{W}}=\left(\alpha \sqrt{r^{\mathcal{W}}}-\beta p^{\mathcal{W}}\right)\left(p^{\mathcal{W}}-\omega^{\mathcal{W}}\right)+V_{R}^{\mathcal{W}}\left(k A^{\mathcal{W}}-\lambda p^{\mathcal{W}} \sqrt{r^{\mathcal{W}}}-\delta r^{\mathcal{W}}\right)
\end{aligned}
$$

are satisfied for any value of $r^{\mathcal{W}}(t) \in(0,1]$. Since we solve the game á la Stakelberg, where $\mathrm{M}$ is the leader. Therefore, we start by solving the $R$ 's optimization problem. The optimization of $R$ 's HJB with respect to the pricing strategy leads to:

$$
p^{\mathcal{W}}=\frac{\omega^{\mathcal{W}} \beta-\sqrt{r^{\mathcal{W}}}\left(\lambda V_{R}^{\mathcal{W}}-\alpha\right)}{2 \beta}
$$


Substituiting Eq. (30) in the M's HJB gives:

$$
\begin{array}{r}
\rho V_{M}^{\mathcal{W}}=\left(\alpha \sqrt{r^{\mathcal{W}}}-\beta \frac{\omega^{\mathcal{W}} \beta-\sqrt{r^{\mathcal{W}}}\left(\lambda V_{R}^{\mathcal{W}}-\alpha\right)}{2 \beta}\right)\left(\frac{\omega^{\mathcal{W}} \beta-\sqrt{r^{\mathcal{W}}}\left(\lambda V_{R}^{\mathcal{W}}-\alpha\right)}{2 \beta}+\Delta \sqrt{r^{\mathcal{W}}}\right)-\frac{\mu\left(A^{\mathcal{W}}\right)^{2}}{2} \\
+V_{M}^{\mathcal{W}}\left(k A^{\mathcal{W}}-\lambda \frac{\omega^{\mathcal{W}} \beta-\sqrt{r^{\mathcal{W}}}\left(\lambda V_{R}^{\mathcal{W}}-\alpha\right)}{2 \beta} \sqrt{r^{\mathcal{W}}}-\delta r^{\mathcal{W}}(\$ 1)\right.
\end{array}
$$

Maximizing with respect to green efforts, $A^{\mathcal{W}}$, and whoselsale price, $\omega^{\mathcal{W}}$, gives:

$$
\begin{aligned}
A^{\mathcal{W}} & =\frac{k V_{M}^{\mathcal{W}}}{\mu} \\
\omega^{\mathcal{W}} & =\frac{\left(\lambda V_{R}^{\mathcal{W},}-\left(\Delta \beta+\lambda V_{M}^{\mathcal{W}}\right)\right)}{\beta} \sqrt{r^{\mathcal{W}}}
\end{aligned}
$$

Plugging Eq. (33) in Eq. (30), we obtain the optimal price:

$$
p^{\mathcal{W}}=\frac{\left(\alpha-\Delta \beta-\lambda V_{M}^{\mathcal{W} \prime}\right)}{2 \beta} \sqrt{r^{\mathcal{W}}}
$$

Substituting the optimal strategies inside Eq. (29) and Eq. (31) and simplyfing we obtain:

$$
\begin{aligned}
4 \beta \mu \rho V_{M}^{\mathcal{W}}= & \mu\left(\alpha+\Delta \beta-\lambda V_{M}^{\mathcal{W} \prime}\right)\left(\alpha+\Delta \beta+\lambda V_{M}^{\mathcal{W} \prime}\right) r^{\mathcal{W}}+2 \beta V_{M}^{\mathcal{W}} k^{2} \\
& +2 \mu V_{M}^{\mathcal{W}}\left(\lambda\left(\Delta \beta-\alpha+\lambda V_{M}^{\mathcal{W} \prime}\right)-2 \beta \delta\right) r^{\mathcal{W}} \\
4 \beta \mu \rho V_{R}^{\mathcal{W}}= & \mu\left(\left(\alpha+\Delta \beta+\lambda V_{M}^{\mathcal{W} \prime}\right)^{2}-4 \alpha \lambda V_{R}^{\mathcal{W}}\right) r+4 \beta V_{R}^{\mathcal{W}}\left(k^{2} V_{M}^{\mathcal{W},}-\delta \mu r^{\mathcal{W}}\right)
\end{aligned}
$$

To solve the previous pair of equations, we can conjecture linear value functions $V_{M}^{\mathcal{W}}=B_{1}^{\mathcal{W}} r \mathcal{W}+B_{2}^{\mathcal{W}}$ and $V_{R}^{\mathcal{W}}=L_{1}^{\mathcal{W}} r^{\mathcal{W}}+L_{2}^{\mathcal{W}}$. Substituting these conjectures and their derivatives inside Eqs $(35)-(36)$ gives:

$$
\begin{aligned}
4 \beta \mu \rho\left(B_{1}^{\mathcal{W}} r^{\mathcal{W}}+B_{2}^{\mathcal{W}}\right)= & \mu\left(\alpha+\Delta \beta-\lambda B_{1}^{\mathcal{W}}\right)\left(\alpha+\Delta \beta+\lambda B_{1}^{\mathcal{W}}\right) r^{\mathcal{W}} \\
& +2 \beta B_{1}^{\mathcal{W}} k^{2}+2 \mu B_{1}^{\mathcal{W}}\left(\lambda\left(\Delta \beta-\alpha+\lambda B_{1}^{\mathcal{W}}\right)-2 \beta \delta\right)^{\mathcal{W}} r \\
4 \beta \mu \rho\left(L_{1}^{\mathcal{W}} r^{\mathcal{W}}+L_{2}^{\mathcal{W}}\right)= & \mu\left(\left(\alpha+\Delta \beta+\lambda B_{1}^{\mathcal{W}}\right)^{2}-4 \alpha \lambda L_{1}^{\mathcal{W}}\right) r^{\mathcal{W}}+4 \beta L_{1}^{\mathcal{W}}\left(k^{2} B_{1}^{\mathcal{W}}-\delta \mu r^{\mathcal{W}}\right)
\end{aligned}
$$


By identification, we obtain the following system of equations:

$$
\begin{aligned}
-4 \beta \mu \rho B_{1}^{\mathcal{W}}+\mu\left(\alpha+\Delta \beta-\lambda B_{1}^{\mathcal{W}}\right)\left(\alpha+\Delta \beta+\lambda B_{1}^{\mathcal{W}}\right)+2 \mu B_{1}^{\mathcal{W}}\left(\lambda\left(\Delta \beta-\alpha+\lambda B_{1}^{\mathcal{W}}\right)-2 \beta \delta\right) & =0 \\
-4 \beta \mu \rho B_{2}^{\mathcal{W}}+2 \beta\left(B_{1}^{\mathcal{W}}\right)^{2} k^{2} & =0 \\
-4 \beta \mu \rho L_{1}^{\mathcal{W}}+\mu\left(\left(\alpha+\Delta \beta+\lambda B_{1}^{\mathcal{W}}\right)^{2}-4 \alpha \lambda L_{1}^{\mathcal{W}}\right)+4 \beta L_{1}^{\mathcal{W}} \delta \mu & =0 \\
-4 \beta \mu \rho L_{2}^{\mathcal{W}}+4 \beta L_{1}^{\mathcal{W}} k^{2} B_{1}^{\mathcal{W}} & =0
\end{aligned}
$$

We can select the negative root of $B_{1}^{\mathcal{W}}$, which is given by

$$
B_{1}^{\mathcal{W}}=\frac{\left((\alpha-\Delta \beta) \lambda+2 \beta(\delta+\rho)-2 \sqrt{\beta(\delta+\rho)((\alpha-\Delta \beta) \lambda+\beta(\delta+\rho))-\Delta \alpha \beta \lambda^{2}}\right)}{\lambda^{2}}
$$

Then, the remaining parameters are given by:

$$
\begin{aligned}
B_{2}^{\mathcal{W}} & =\frac{\left(B_{1}^{\mathcal{W}}\right)^{2} k^{2}}{2 \mu \rho} \\
L_{1}^{\mathcal{W}} & =\frac{\mu\left(\alpha+\Delta \beta+\lambda B_{1}^{\mathcal{W}}\right)^{2}}{4 \mu(\alpha \lambda-\beta(\delta+\rho))} \\
L_{2}^{\mathcal{W}} & =\frac{L_{1}^{\mathcal{W}} B_{1}^{\mathcal{W}} k^{2}}{\mu \rho}
\end{aligned}
$$

Proof. of Corollary 1. The results in Corollary 1 follow the following derivatives:

- $\frac{\partial A_{S S}^{\mathcal{R}}}{\partial \phi}=\frac{k}{\mu} \frac{\partial B_{1}^{\mathcal{R}}}{\partial \phi}>0$

• $\frac{\partial r_{S S}^{\mathcal{R}}}{\partial \phi}=\left\{2 \beta k^{2}\left(-B_{1}^{\mathcal{R}}+\frac{\partial B_{1}^{\mathcal{R}}}{\partial \phi}(1-\phi)\right) D E N\left[r_{S S}^{\mathcal{R}}\right]+\mu N U M\left[r_{S S}^{\mathcal{R}}\right]\left((\alpha \lambda+2 \beta \delta)+\lambda^{2} \frac{\partial L_{1}^{\mathcal{R}}}{\partial \phi}\right)\right\} / D E N\left[r_{S S}^{\mathcal{R}}\right]^{2}>$

0

• $\frac{\partial p_{S S}^{\mathcal{R}}}{\partial \phi}=\frac{\left[\left(-\alpha-\frac{\partial L_{1}^{\mathcal{R}}}{\partial \phi} \lambda\right) \sqrt{r}+\left(\alpha(1-\phi)-\lambda L_{1}^{\mathcal{R}}\right) \sqrt{\frac{\partial r_{S S}^{\mathcal{R}}}{\partial \phi}}\right] D E N\left[p_{S S}^{\mathcal{R}}\right]+2 \beta N U M\left[p_{S S}^{\mathcal{R}}\right]}{[2 \beta(1-\phi)]^{2}}>0$

- $\frac{\partial D_{S S}^{\mathcal{R}}}{\partial \phi}=\alpha \sqrt{\frac{\partial r_{S S}^{\mathcal{R}}}{\partial \phi}}-\beta \frac{\partial p_{S S}^{\mathcal{R}}}{\partial \phi}>0 ;$

$\downarrow \frac{\partial V_{M_{S S}^{\mathcal{R}}}}{\partial \phi}=\frac{\partial B_{1}^{\mathcal{R}}}{\partial \phi} r_{S S}^{\mathcal{R}}+\frac{\partial r_{S S}^{\mathcal{R}}}{\partial \phi} B_{1}^{\mathcal{R}}+\frac{\partial B_{2}^{\mathcal{R}}}{\partial \phi}>0$

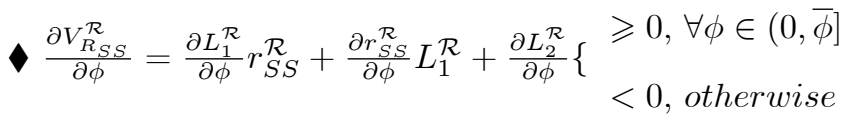

Proof. of Proposition 2. We search for a pair of bounded and continuously differentiable value functions 
$V_{M}^{\mathcal{R}}\left(r^{\mathcal{R}}\right)$ and $V_{R}^{\mathcal{R}}\left(r^{\mathcal{R}}\right)$ for which a unique solution for $r^{\mathcal{R}}(t)$ does exist, and the HJB equations:

$$
\begin{aligned}
\rho V_{M}^{\mathcal{R}} & =\left(\alpha \sqrt{r^{\mathcal{R}}}-\beta p^{\mathcal{R}}\right)\left(p^{\mathcal{R}} \phi+\Delta \sqrt{r^{\mathcal{R}}}\right)-\frac{\mu\left(A^{\mathcal{R}}\right)^{2}}{2}+V_{M}^{\mathcal{R}}\left(k A^{\mathcal{R}}-\lambda p^{\mathcal{R}} \sqrt{r^{\mathcal{R}}}-\delta r^{\mathcal{R}}\right) \\
\rho V_{R}^{\mathcal{R}} & =\left(\alpha \sqrt{r^{\mathcal{R}}}-\beta p^{\mathcal{R}}\right) p^{\mathcal{R}}(1-\phi)+V_{R}^{\mathcal{R},}\left(k A^{\mathcal{R}}-\lambda p^{\mathcal{R}} \sqrt{r^{\mathcal{R}}}-\delta r^{\mathcal{R}}\right)
\end{aligned}
$$

are always satisfied for any value of $r^{\mathcal{R}}(t) \in(0,1]$. Since we solve the game á la Stakelberg, where $M$ is the leader. Nevertheless, the pricing and green efforts strategies are independent; therefore, solving the Stakelberg game corresponds to solving the Nash game. In fact, the firms reaction functions are given by

$$
\begin{aligned}
& \frac{\partial V_{M}^{\mathcal{R}}}{\partial A^{\mathcal{R}}}=k V_{M}^{\mathcal{R},}-A^{\mathcal{R}} \mu \\
& \frac{\partial V_{R}^{\mathcal{R}}}{\partial p^{\mathcal{R}}}=p^{\mathcal{R}} \beta \phi-p^{\mathcal{R}} \beta-\sqrt{r^{\mathcal{R}}} \lambda V_{R}^{\mathcal{R},}+(1-\phi)\left(\sqrt{r^{\mathcal{R}}} \alpha-p^{\mathcal{R}} \beta\right)
\end{aligned}
$$

Therefore, the optimal strategies result as follows:

$$
\begin{aligned}
A^{\mathcal{R}} & =\frac{k V_{M}^{\mathcal{R}}}{\mu} \\
p^{\mathcal{R}} & =\frac{\alpha(1-\phi)-\lambda V_{R}^{\mathcal{R}},}{2 \beta(1-\phi)} \sqrt{r^{\mathcal{R}}}
\end{aligned}
$$

Substituiting the optimal strategies inside the firms' HJBs gives:

$$
\begin{aligned}
4 \mu \beta(1-\phi)^{2} \rho V_{M}^{\mathcal{R}}= & \mu\left((1-\phi)(2 \Delta \beta+\alpha \phi)-\lambda \phi V_{R}^{\mathcal{R} \prime}\right)\left(\alpha(1-\phi)+\lambda V_{R}^{\mathcal{R} \prime}\right) r^{\mathcal{R}}+2 \mu(1-\phi) V_{M}^{\mathcal{R} \prime} \\
& \left(-\lambda\left(\alpha(1-\phi)-\lambda V_{R}^{\mathcal{R} \prime}\right)-2 \beta \delta(1-\phi)\right) r^{\mathcal{R}}+2 \beta(1-\phi)^{2} k^{2} V_{M}^{\mathcal{R}, 2} \\
4(1-\phi) \beta \mu \rho V_{R}^{\mathcal{R}}= & \mu\left(\alpha \phi-\alpha+\lambda V_{R}^{\mathcal{R} \prime}\right)^{2} r^{\mathcal{R}}+4(1-\phi) \beta V_{R}^{\mathcal{R} \prime}\left(k^{2} V_{M}^{\mathcal{R},}-\delta \mu r^{\mathcal{R}}\right)
\end{aligned}
$$

To solve the previous pair of equations, we can conjecture linear value functions $V_{M}^{\mathcal{R}}=B_{1}^{\mathcal{R}} r^{\mathcal{R}}+B_{2}^{\mathcal{R}}$ and $V_{R}^{\mathcal{R}}=L_{1}^{\mathcal{R}} r^{\mathcal{R}}+L_{2}^{\mathcal{R}}$. Substituting these conjectures and their derivatives inside Eqs $(53)-(54)$ gives:

$$
\begin{aligned}
4 \mu \beta(1-\phi)^{2} \rho\left(B_{1}^{\mathcal{R}} r^{\mathcal{R}}+B_{2}^{\mathcal{R}}\right)= & \left.\mu\left((1-\phi)(2 \Delta \beta+\alpha \phi)-\lambda \phi L_{1}^{\mathcal{R}}\right)\left(\alpha(1-\phi)+\lambda L_{1}^{\mathcal{R}}\right) r^{\mathcal{R}}+2 \mu(1-\phi) \beta^{\mathcal{R}} 5\right) \\
& \left(-\lambda\left(\alpha(1-\phi)-\lambda L_{1}^{\mathcal{R}}\right)-2 \beta \delta(1-\phi)\right) r+2 \beta(1-\phi)^{2} k^{2}\left(B_{1}^{\mathcal{R}}\right)^{2} \\
4(1-\phi) \beta \mu \rho\left(L_{1}^{\mathcal{R}} r^{\mathcal{R}}+L_{2}^{\mathcal{R}}\right)= & \mu\left(\alpha \phi-\alpha+\lambda L_{1}^{\mathcal{R}}\right)^{2} r^{\mathcal{R}}+4(1-\phi) \beta L_{1}^{\mathcal{R}}\left(k^{2} B_{1}^{\mathcal{R}}-\delta \mu r^{\mathcal{R}}\right)
\end{aligned}
$$

By identification, the model parameters are: 


$$
\begin{aligned}
-4 \mu \beta(1-\phi)^{2} \rho B_{1}^{\mathcal{R}}+\mu\left((1-\phi)(2 \Delta \beta+\alpha \phi)-\lambda \phi L_{1}^{\mathcal{R}}\right)\left(\alpha(1-\phi)+\lambda L_{1}^{\mathcal{R}}\right) & \\
+2 \mu(1-\phi) B_{1}^{\mathcal{R}}\left(-\lambda\left(\alpha(1-\phi)-\lambda B_{1}^{\mathcal{R}}\right)-2 \beta \delta(1-\phi)\right) & =0 \\
-4 \mu \beta(1-\phi)^{2} \rho B_{2}^{\mathcal{R}}+2 \beta(1-\phi)^{2} k^{2}\left(B_{1}^{\mathcal{R}}\right)^{2} & =0 \\
-4(1-\phi) \beta \mu \rho L_{1}^{\mathcal{R}}+\mu\left(\alpha \phi-\alpha+\lambda L_{1}^{\mathcal{R}}\right)^{2}-4 \delta \mu(1-\phi) \beta L_{1}^{\mathcal{R}} & =0 \\
-4(1-\phi) \beta \mu \rho B_{2}^{\mathcal{R}}+4(1-\phi) \beta L_{1}^{\mathcal{R}} k^{2} B_{1}^{\mathcal{R}} & =0
\end{aligned}
$$

We can see that there exists one solution only for $B_{1}^{\mathcal{R}}$ while we take the negative root for $R_{1}^{\mathcal{R}}$. The solution is given as follows:

$$
\begin{aligned}
B_{1}^{\mathcal{R}} & =\frac{\mu\left(\lambda L_{1}^{\mathcal{R}}+\alpha(1-\phi)\right)\left((2 \Delta \beta+\alpha \phi)(1-\phi)-\lambda \phi L_{1}^{\mathcal{R}}\right)}{2 \mu(1-\phi)\left((\lambda \alpha+2 \beta(\delta+\rho))(1-\phi)-\lambda^{2} L_{1}^{\mathcal{R}}\right)} \\
B_{2}^{\mathcal{R}} & =\frac{k^{2}\left(B_{1}^{\mathcal{R}}\right)^{2}}{2 \mu \rho} \\
L_{1}^{\mathcal{R}} & =\frac{(\alpha \lambda+2 \beta(\delta+\rho))(1-\phi)-2 \sqrt{\beta(1-\phi)^{2}(\delta+\rho)(\alpha \lambda+\beta(\delta+\rho))}}{\lambda^{2}} \\
L_{2}^{\mathcal{R}} & =\frac{k^{2} B_{1}^{\mathcal{R}} L_{1}^{\mathcal{R}}}{\mu \rho}
\end{aligned}
$$

\subsection{Pareto analysis on different sets}

Hereby, we carry out the Pareto analysis on two different parameter sets to demonstrate the robustness of our findings. Specifically, we use the following two parameter sets:

- high parameter values, by fixing the parameters as follows: $\alpha=3, \beta=0.8, k=0.15, \Delta=$ $0.3, \delta=0.3, \rho=0.15$ and $\mu=1.5$

- low parameter values, by fixing the parameters as follows: $\alpha=1, \beta=0.4, k=0.05, \Delta=$ $0.1, \delta=0.1, \rho=0.05$ and $\mu=0.5$.

As we display in Figures A1 and A2, the findings that we obtain in Figure 10 are confirm when taking different parameter sets. 


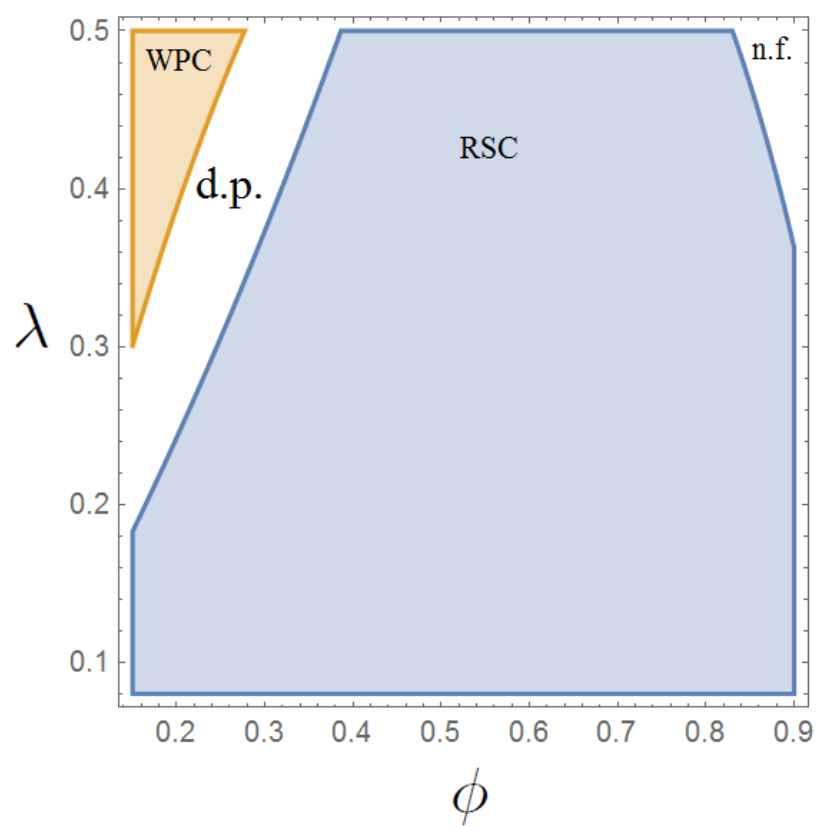

Figure A1 - Pareto improving region with high parameter values

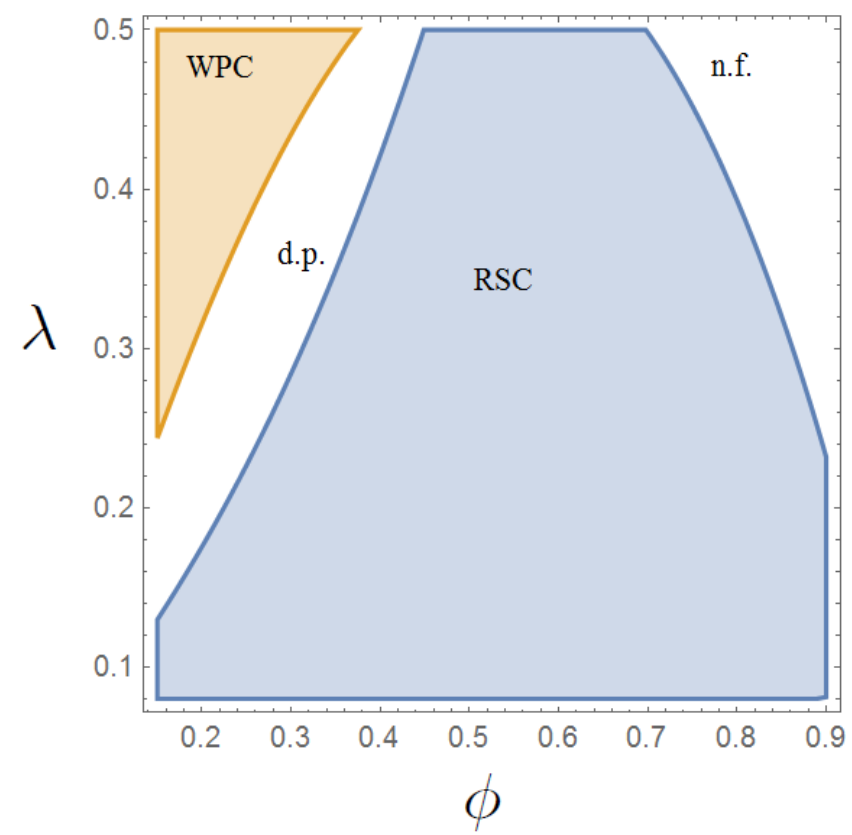

Figure A2 - Pareto improving region with low parameter values 


\section{References}

[1] Agrawal, V., \& Tokay, L. B. (2010). Interdisciplinarity in closed-loop supply chain management research. In F. E. Ferguson \& G. C. Souza (Eds.), Closed-loop supply chains: new developments to improve the sustainability of business practices. Boca Raton: CRC Press

[2] Cachon, G. P. (2003). Supply chain coordination with contracts. In S. Graves \& T. de Kok (Eds.), Handbooks in operations research and management science: supply chain management. Amsterdam: North- Holland.

[3] Cachon, G. P., \& Lariviere, M. A. (2005). Supply chain coordination with revenue-sharing contracts: strengths and limitations. Management science, 51(1), 30-44.

[4] De Giovanni, P. (2014), 'Environmental collaboration in a closed-loop supply chain with a reverse revenue sharing contract', Annals of Operations Research 220(1), 135-157.

[5] De Giovanni, P. (2017). Closed-loop supply chain coordination through incentives with asymmetric information. Annals of Operations Research, 253(1), 133-167.

[6] De Giovanni, P.; Reddy, P. V. \& Zaccour, G. (2016), 'Incentive strategies for an optimal recovery program in a closed-loop supply chain', European Journal of Operational Research 249(2), 605-617.

[7] De Giovanni, P. \& Zaccour, G. (2013), Cost-Revenue Sharing in a Closed-Loop Supply Chain'Advances in Dynamic Games', Annals of the International Society of Dynamic Games, 12, 395-421.

[8] De Giovanni, P. \& Zaccour, G. (2014), 'A two-period game of a closed-loop supply chain', European Journal of Operational Research 232(1), 22-40.

[9] De Giovanni, P. \& Ramani, V. (2018), 'Product cannibalization and the effect of a service strategy', Journal of the Operational Research Society, 1-17.

[10] De Giovanni, P., \& Zaccour, G. (2018). Optimal quality improvements and pricing strategies with active and passive product returns. Omega. in press.

[11] Dockner, E. J., Jorgensen, S., Van Long, N., \& Sorger, G. (2000). Differential games in economics and management science. Cambridge University Press.

[12] Fleischmann, M., Bloemhof-Ruwaard, J., Dekker, R., Van der Laan, E., van Nunen, J., \& Van Wassenhove, L. (1997). Quantitative models for reverse logistics. European journal of operational research, 103, $1-17$. 
[13] Guide Jr, V. D. R. \& Van Wassenhove, L. N. (2009), 'OR FORUM-The evolution of closed-loop supply chain research', Operations research 57(1), 10-18.

[14] Miao, Z., Fu, K., Xia, Z., \&Wang, Y. (2017). Models for closed-loop supply chain with trade-ins. Omega, $66,308-326$.

[15] Minner, S. \& Kleber, R. (2001), 'Optimal control of production and remanufacturing in a simple recovery model with linear cost functions', OR-Spektrum 23(1), 3-24.

[16] Ramani, V. \& De Giovanni, P. (2017), 'A two-period model of product cannibalization in an atypical Closed-loop Supply Chain with endogenous returns: The case of DellReconnect', European Journal of Operational Research 262(3), 1009-1027.

[17] Savaskan, R. C.; Bhattacharya, S. \& Van Wassenhove, L. N. (2004), 'Closed-loop supply chain models with product remanufacturing', Management science 50(2), 239-252.

[18] Sethi, S. P., \& Thompson, G. L. (2000). Optimal control theory: applications to management science and economics. Springer.

[19] Sethi, S. P. (1983). Deterministic and stochastic optimization of a dynamic advertising model. Optimal Control Applications and Methods, 4(2), 179-184.

[20] Zhou, W., Zheng, Y., \& Huang, W. (2017). Competitive advantage of quali. . . ed WEEE recyclers through EPR legislation. European Journal of Operational Research, 257(2), 641-655. 